\title{
La vie de Gregorio Lòpez par Thomas White (1592-1676), ou la sanctification d'un ermite hétérodoxe par un catholique hérétique
}

The Life Of Gregorio Lòpez by Thomas White (1592-1676), or the Sanctification of a Heterodox Hermit by a Heretical Catholic

Thérèse-Marie Jallais

\section{OpenEdition Journals}

Édition électronique

URL : http://journals.openedition.org/rfcb/3571

DOI : 10.4000/rfcb.3571

ISSN : 2429-4373

Éditeur

CRECIB - Centre de recherche et d'études en civilisation britannique

Édition imprimée

Date de publication : 1 mars 2013

ISBN : 2-911580-37-0

ISSN : 0248-9015

Référence électronique

Thérèse-Marie Jallais, «La vie de Gregorio Lòpez par Thomas White (1592-1676), ou la sanctification d'un ermite hétérodoxe par un catholique hérétique », Revue Française de Civilisation Britannique [En ligne], XVIII-1 | 2013, mis en ligne le 01 mars 2013, consulté le 22 mars 2020. URL : http:// journals.openedition.org/rfcb/3571 ; DOI : https://doi.org/10.4000/rfcb.3571

Ce document a été généré automatiquement le 22 mars 2020.

Revue française de civilisation britannique est mis à disposition selon les termes de la licence Creative Commons Attribution - Pas d'Utilisation Commerciale - Pas de Modification 4.0 International. 


\section{La vie de Gregorio Lòpez par Thomas White (1592-1676), ou la sanctification d'un ermite hétérodoxe par un catholique hérétique}

The Life Of Gregorio Lòpez by Thomas White (1592-1676), or the Sanctification of a Heterodox Hermit by a Heretical Catholic

Thérèse-Marie Jallais

Notre propos n'est pas ici de déterminer ce qu'est un saint, mais d'expliquer l'engouement éditorial suscité en terre anglaise par un modèle de piété catholique : Gregorio López (1542-1596). L'homme ne fut jamais canonisé. Et pourtant ! Ce laïc espagnol devint un parangon de vertu, et les biographies qui lui furent consacrées rencontrèrent un vif succès, dépassant très largement le cadre du milieu où il vécut : le désert mexicain. La Vida que hizo el siervo de Dios Gregorio Lopez (1613) connut, en effet, de nombreuses traductions en Europe ${ }^{1}$. Ce catholique aurait pu demeurer un exemple de piété vénéré localement, comme il était fréquent à l'époque baroque ${ }^{2}$. Comment, alors, rendre compte de cette renommée? Peut-on y voir la force de la dévotion populaire, qui, contre le choix des instances de l'Église catholique, aurait réussi à imposer ses propres idéaux en matière d'incarnation de la perfection, idéaux parfois fort distants de ceux des théologiens experts en orthodoxie de la congrégation des rites, qui, seule, pouvait sanctifier? En partie peut-être. Mais le rayonnement de cette figure en Europe, dans des cercles ou des groupes de théologiens chevronnés, pourrait indiquer d'autres pistes. Pourquoi cette figure fut-elle si bien accueillie dans les milieux protestants? Pourquoi son succès se confirma-t-il, en Angleterre, jusqu'au milieu du XVII ${ }^{e}$ siècle ? Ce saint non estampillé y fut-il l'objet, dans le contexte de la Contre-Réforme, de ce que l'on pourrait appeler une récupération théologique? Si tel était le cas, quels pouvaient en être les enjeux? Sinon, d'où vient ce succès d'édition? 
2 Deux précisions s'imposent en préambule : l'histoire de la spiritualité ne constitue-en rien un monde clos. Dans la relation entre la représentation sociale du saint et la doctrine catholique se font jour, au XVII ${ }^{e}$ siècle, des changements visant à redéfinir la pratique hagiographique à l'intérieur d'une dynamique théologique très tendue. À l'âge baroque, en effet, ces vies de saints étaient l'une des manifestations les plus visibles des divergences entre catholiques et protestants, le saint canonisé représentant pour les réformés une métaphore, sinon la métaphore privilégiée du corps des controverses et, en particulier, des désaccords qui opposaient les deux confessions chrétiennes sur la conception même de la Tradition de l'Église ${ }^{3}$. Rappelons simplement que, pour Luther, nul n'était besoin d'intercesseur, hormis le Christ : la foi seule justifiait, au point que le culte des saints participait de l'idolâtrie et mettait en danger le monothéisme. Jean Calvin (1509-1564) consomma définitivement la rupture avec la conception catholique du saint en posant que le culte des reliques et des images attribuait à Dieu une apparence visible, ce qui était inacceptable sur le plan du dogme $e^{4}$. L'idée que des chrétiens puissent être des modèles sur la voie de la perfection n'est, certes, pas absente de la culture protestante, mais ils ne sauraient avoir le statut d'intermédiaire. En second lieu, il convient de rappeler que la théologie fut, comme tous les domaines $\mathrm{du}$ savoir au XVII ${ }^{\mathrm{e}}$ siècle, l'objet de divergences épistémologiques, de redécoupages de ses propres champs de connaissance. La science religieuse se transforma : à l'instar des autres disciplines, elle subit l'influence du rationalisme ambiant, qui se traduisit par un souci de soumettre ses textes à l'épreuve des preuves historiques et par une soif de vérification érudite que formalisa l'exégèse ${ }^{5}$. Ces deux niveaux d'analyse - théologique et scientifique - ne s'emboîtent pas à la manière de poupées gigognes, pas plus qu'ils ne se trouvent liés par des relations de cause à effet. Les temps ne sont pas parfaitement synchroniques, les espaces mêmes ne se recoupent pas harmonieusement. Ni temps de l'étale, ni temps du reflux, la spiritualité des saints est source de vives controverses entre 1560 et 1680 .

\section{Du peuple politique au peuple de la Tradition de l'Église}

3 La première traduction anglaise de La Vida que hizo el siervo de Dios Gregorio López en algunos lugares de esta nueva Espana, intitulée The Life of Gregorio López fut publiée à Paris, en $1638^{6}$. Elle était signée ' $N$. N.', le traducteur ayant préféré garder l'anonymat. Derrière ces fausses initiales se cache Thomas White (1592/3-1676), prêtre catholique anglais lié à Port-Royal, et détenteur de multiples pseudonymes parmi lesquels "Albius», «Blacklow» et «Blacklaw »7. Jusqu'aux années 1990, l'homme restait mal connu. Mais les travaux de Bruno Neveu, de Beverley Southgate et, plus récemment, ceux de Stefania Tutino ont éclairé la personnalité et le rôle intellectuel de Thomas White, tant dans le domaine de la pensée politique et scientifique que dans celui de la théologie ${ }^{8}$.

L'homme avait été éduqué à l'université catholique de Douai, où il fut ordonné prêtre en 1617, puis nommé enseignant en 1622. En 1650, il en fut le vice-président. Après des séjours nombreux à Paris, à Saint-Omer, à Séville, et à Valladolid, il devint, entre 1625 et 1629 , l'agent des catholiques anglais à Rome, au moment où le mariage de Charles $1^{\text {er }}$ Stuart et Henriette-Marie de France pouvait faire espérer un retour du catholicisme en Angleterre. À partir des années 1640, White fréquenta assidûment le cercle des 
scientifiques parisiens de Mersène, rencontra Gassendi, Descartes et des membres de la Royal Society. Dans Peripateticall Institutions (1647), ouvrage de physique, il parvenait à réconcilier approche aristotélicienne et philosophie moderne en acceptant l'héliocentrisme de Copernic sans renoncer à l'idée d'un centre incluant la terre, cœur de l'immensité cosmique'. Sa réflexion politique, d'une grande originalité et d'une remarquable indépendance, exerça une réelle influence sur nombre de ses contemporains, sur Thomas Hobbes, par exemple ${ }^{10}$. Celui que Descartes surnommait "Vitus » était considéré comme "l'un des philosophes les plus subtils de son temps »"11. Dans The Grounds of Obedience and Government (1655), il avait, de manière inattendue, affirmé que l'opposition à Charles $1^{\text {er }}$ était fondée, et que les catholiques devaient accepter l'autorité de Cromwell ${ }^{12}$. Cette position lui valut d'être rejeté par un certain nombre de catholiques anglais, pour qui l'appartenance à l'Église catholique romaine allait de pair avec le soutien aux Stuart ${ }^{13}$. Dans ce même ouvrage, il défendait clairement le concept de souveraineté populaire et affirmait que tout régime politique devait se donner pour objectif premier le bien-être du peuple, ce en quoi il différait du Leviathan de Hobbes comme de l'Oceana de Harrington ${ }^{14}$. L'insistance sur cette notion entendue dans son acception moderne se retrouve dans ses traités de théologie. L'Église ne peut définir la Tradition; elle ne peut qu'en témoigner ${ }^{15}$. Cette Tradition inclut le peuple de Dieu, qui participe de la réception orale des Écritures. Selon Thomas White, cette écoute peut bien sûr faire l'objet d'erreurs, mais celles-ci ne relèvent en rien de l'hérésie, précisément parce qu'il s'agit d'une transmission orale. L'hérésie ne peut être le fait que des théologiens spécialistes des écrits bibliques et de leurs interprétations, ceci tout au long de l'histoire de l'Église ${ }^{16}$. Avec subtilité, le théologien anglais fait donc éclater la dichotomie rigide des discours savants sur les concepts d'hérésie et d'orthodoxie en lui substituant un autre couple dialectique, celui qui oppose la Parole reçue oralement par le peuple de Dieu à l'ensemble des traditions écrites, c'est-à-dire exégétiques ${ }^{17}$.

5 Ces analyses politico-religieuses firent de White le porte-parole reconnu d'un groupe: les Blackloists. Henry Holden, John Sergeant et Kenelm Digby, qui en étaient avec lui les chefs de file, élaborèrent en 1647 un programme à la théologie révolutionnaire intitulé le Holden Plan: l'Église catholique, en Angleterre, devait s'engager à renoncer à tout pouvoir temporel ; en contrepartie, le Parlement lui garantirait la liberté de culte ${ }^{18}$. La nomination de six ou sept évêques catholiques par ce même Parlement assurerait la reconnaissance officielle de l'Église romaine ${ }^{19}$. Cromwell soutenait le projet, mais Rome, sans surprise, le condamna en 1655, et Thomas White fut contraint à la Restauration de fuir aux Pays-Bas ${ }^{20}$. S'engagea alors une guerre ouverte entre les jésuites, défenseurs de l'autorité pontificale, et les partisans du Holden Plan. Ces derniers furent accusés de jansénisme: accusation fondée que White ne démentit pas, tout au contraire, car les relations entre les Blackloists et Port-Royal étaient étroites et régulières ${ }^{21}$. L'opposition à l'ultramontanisme, la remise en cause de l'autorité pontificale, la méfiance extrême à l'égard de la Compagnie de Jésus, dont les pratiques tendaient à nier la hiérarchie épiscopale au profit d'un pouvoir confisqué par des réguliers, réunissaient les partisans de White et les jansénistes français. 


\section{La mystique rhéno-flamande du « saint »}

6 Lorsque la traduction qui retient notre attention est publiée, en 1638, les relations des Blackloists avec Rome ne sont pas rompues, et leurs positions politiques ne sont pas encore ouvertement affichées. Un point surprend: Thomas White, écrivain prolifique, maîtrisant couramment plusieurs langues, dont l'espagnol, le français et l'italien, ne faisait pas de la traduction son activité principale. Parmi tous les ouvrages dont nous savons qu'il est l'auteur - une cinquantaine environ - ne figure qu'une seule autre traduction ${ }^{22}$. La Vida que hizo el siervo de Dios Gregorio López en algunos lugares de esta nueva Espana présentait à ses yeux des qualités particulières, comme nous le montrerons par la suite. Toutefois, la médiocre qualité littéraire de l'ouvrage espagnol et son absence de réflexion théologique originale expliquent mal a priori l'intérêt du subtil théologien pour une œuvre qui, sur ces deux plans, peut être qualifiée de parfaitement mineure. En réalité, la traduction de Thomas White relève de l'adaptation libre, des coupes très importantes étant opérées dans le texte de 1613. Cette pratique était très répandue à une époque où les droits d'auteur n'existaient pas. On la retrouve au siècle suivant chez le méthodiste John Wesley, qui, dans les ouvrages qu'il destinait à ses lecteurs anglophones, n'hésitait pas à traduire et à adapter les écrits de catholiques français dont il taisait le nom. ${ }^{23}$ Dans ce contexte, si la question de la fidélité à l'ouvrage originel ne présente guère d'intérêt, la mise en lumière de la conception de la spiritualité que Thomas White s'attache à développer pour son lectorat anglophone - catholique ou protestant - doit permettre de mieux cerner comment s'élabore, sous la plume du traducteur-adaptateur, une image de saint originale qui soit acceptable en terre puritaine.

7 De Gregorio López, on ne sait presque rien, remarque Thomas White. Son enfance reste obscure. Il aurait été élevé à la cour d'Espagne, aurait peut-être même été un fils illégitime de Philippe II ! ${ }^{24}$ On retrouve ici un lieu commun de la figure du saint véhiculé par la tradition hagiographique médiévale : ses origines se devaient d'être nobles. De nombreuses spéculations circulaient sur son appartenance religieuse : il aurait été juif ou... luthérien ${ }^{25}$. Un point reste certain : son départ pour le Mexique, à 20 ans, en 1562. Selon Thomas White, López aurait entretenu au Nouveau-Mexique des rapports tendus avec la hiérarchie catholique acquise à une discipline cénobitique : adepte de la vie en communauté, elle regardait d'un mauvais œil tous ceux qui chérissaient l'érémitisme. Le problème perdura, car l'homme ne fréquentait guère les lieux de culte, préférant la solitude pour cultiver une dévotion tout intérieure. La Cathédrale de Mexico ordonna une enquête sur ce catholique qui semblait faire fi des sacrements et des règles d'un ordre religieux ${ }^{26}$. López préférait, aux rituels de l'Église, les formes d'adoration silencieuses. La contemplation et la solitude constituaient, selon lui, les seules marques d'une vraie foi, Dieu ne pouvant être atteint que par un acte de volonté, et non par les voies conceptuelles et discursives auxquelles l'Espagnol avait complètement renoncé. Exercer sa volonté pour se plier à la volonté divine afin de connaître l'anéantissement en Dieu : tel était son objectif ${ }^{27}$. Thomas White, le théologien catholique, présente donc un parcours de l'âme dans l'ascèse qui n'est pas sans rappeler un texte contemporain de La Vida intitulé La reigle de perfection, de Benoît de Canfeld, dont la version italienne fut d'ailleurs condamnée par Rome ${ }^{28}$. Ces deux œuvres, en effet, prônent une spiritualité tournée vers l'abstrait, le chrétien établissant un rapport avec l'essence divine sans poser d'intermédiaire, dans une fusion transcendant la réalité du Christ. La spiritualité 
de l'Espagnol s'apparente également à la mystique rhéno-flamande, qui posait que l'âme, en sa pointe extrême, appelée son essence, pouvait parvenir à cette union avec Dieu - union souvent réservée aux mystiques, dont la position se trouvait singulièrement sacralisée ${ }^{29}$. Il convient de noter que le rationalisme cartésien réfuta violemment ce modèle ontologique en posant que l'être suprême restait hors du sujet croyant. Dans cette seconde cartographie de la vie spirituelle qu'illustrait Bossuet, la foi devait être gérée par la raison, qui transformait le sentiment d'amour par la connaissance de la figure incontournable du Christ. Ces deux conceptions de la vie spirituelle s'opposèrent dans la première moitié du XVII ${ }^{e}$ siècle. L'adaptation de Thomas White appartient bien au premier courant, auquel furent particulièrement réceptifs les milieux réformés en Europe, mais dont se détournèrent les milieux catholiques orthodoxes.

Sur le plan didactique, la traduction anglaise remplit parfaitement la première fonction attendue de toute œuvre hagiographique : celle d'enseigner les vertus, en particulier la chasteté, l'humilité, c'est-à-dire la pauvreté, et la dévotion ${ }^{30}$. Mais, du contenu des prières de l'ermite, nous ignorons tout; nous savons seulement qu'elles étaient fort nombreuses. Le théologien White insiste surtout sur l'humilité du chrétien qui, toute sa vie, vécut dans un complet dénuement, le désert mexicain constituant clairement un lieu idéal pour une vie sans attaches matérielles. $\mathrm{Nu}$ - pieds, sans chemise, un sac ceint autour des hanches, il ne mangeait que du maïs, jamais de viande. Hormis la nature de la graminée, toutes les composantes de la convention littéraire valorisant le nécessaire détachement des biens matériels sont présentes ${ }^{31}$. De fait, ce topos indispensable à la construction romanesque du vrai saint s'y déploie sur le modèle de la vie des Pères du désert que valorisait également la tradition catholique janséniste française. Dans le cas de The Life of Gregorio López, la vertu de pauvreté correspond au tableau idéal d'une vie exemplaire brossé dans les textes arétologiques de référence sur la question, en particulier le récit de la vie d'Antoine par Athanase, que le texte anglais, dans l'ordonnancement des divers épisodes, copie parfaitement, au point que l'on pourrait aller jusqu'à dire que, plus la vie du héros espagnol imite celle d'Antoine, plus elle confine à la perfection ${ }^{32}$. Vivre en saint, c'est d'abord partir sur les traces de ce modèle que la lettre du texte se doit, avec subtilité, de mettre en évidence. Enfin, force est de constater la remarquable harmonie entre la mise en œuvre de cette vertu de pauvreté et le milieu socio-culturel dans lequel elle se manifeste. Est ainsi posée une adéquation non problématique entre la fidélité à un message évangélique, sa mise en œuvre, et les valeurs de la société mexicaine.

\section{Le respect de la tradition hagiographique médiévale}

9 Au niveau narratologique, cet apprentissage de la sainteté eût été fort aride s'il avait été présenté comme une simple liste de vertus et de vérités. Thomas White s'inscrit, sur ce point précis, dans la plus pure tradition littéraire catholique : il convenait, en effet, comme le rappelait le jésuite Alonso Ramos, spécialiste du genre, que le récit gardât sa saveur, la dimension ludique et divertissante servant à maintenir l'attention du lecteur ${ }^{33}$. En d'autres termes, ces traités de théologie sous forme narrative se devaient d'être agrémentés d'épisodes où s'abolissait complètement la frontière entre réalité et fiction, où se voyaient minutieusement dépeintes des anecdotes fabuleuses ou extraordinaires, éléments qui soulignent la parenté directe entre ce type de 
construction et les récits épiques du Moyen Âge, dont il était largement inspiré. The Life of Gregorio López se rattache à cette ancienne tradition, tout comme le texte espagnol de 1613. Des personnages merveilleux aident l'ermite à cultiver son petit jardin, la frontière avec le surnaturel étant abolie ${ }^{34}$. López dialogue fréquemment avec les anges et mène des combats spirituels répétés contre des démons, entités matérielles qui l'agressent physiquement, mais qu'il finit toujours par vaincre ${ }^{35}$. Le héros espagnol n'a rien du fondateur ou du pasteur : c'est seulement un vaillant guerrier en lutte contre le diable en son désert.

10 Le cadre de vie et la vie intérieure de Gregorio López sont donc peuplés de personnages surnaturels, de monstres, d'anges et de démons. Si ces derniers renvoient clairement à des épisodes bibliques précis, ce n'est pas le cas des-premiers. Et pourtant ! Leur inscription dans le récit autorise un flou subtil entre surnaturel et réel. En tout état de cause, l'adaptation de Thomas White obéit parfaitement aux lois du genre, dont une des composantes littéraires attendues et incontournables est précisément la présence du merveilleux. L'ermite possède également un don, topos de cette même tradition hagiographique : il est guérisseur et... auteur d'un ouvrage sur la guérison par les plantes ${ }^{36}$. Entre charisme et science, les phénomènes de porosité sont multiples. Sur ce point précis, probablement pour ménager la sensibilité des lecteurs de tradition protestante, aucune référence n'est faite aux effets des charismes, c'est-à-dire aux miracles. Le mot n'est jamais mentionné : 'Healing' peut être entendu au sens littéral ou métaphorique, les deux sens se recouvrant parfaitement. Il est inutile de préciser si l'Espagnol soigne les corps ou les âmes : il soigne, et cela suffit. Du récit reconstruit par Thomas White se dégage une poésie qui prend sa source dans ce mélange savamment dosé entre temps chronologique (celui du travail du héros) et temps de l'extraordinaire, où l'imaginaire peut à loisir se déployer. Les manifestations de la puissance de Dieu relèvent d'une vraie poétique, et expriment la jouissance festive que procurent au lecteur le merveilleux et le surnaturel.

11 The Life of Gregorio López ne saurait être étudiée en fonction de son authenticité ou de sa valeur historique. L'adaptation de Thomas White rattache l'œuvre anglaise à la grande tradition des vies édifiantes dont l'objet consistait à insister sur ce qui était exemplaire, en particulier les vertus du héros, et, ensuite, à structurer les manifestations divines par leur mise en forme narrative. À ces deux niveaux, elle constitue bien la cristallisation littéraire des perceptions et des attentes des lecteurs imprégnés de tradition hagiographique médiévale. Il convient cependant de noter que, du point de vue historiographique, la transition entre ce type de littérature pieuse cultivant toujours le sensationnel et l'extraordinaire et les biographies savantes des modèles de perfection eut précisément lieu entre 1613, date de la première publication de La Vida, et le milieu du XVII ${ }^{e}$ siècle. Cette période vit se développer l'exégèse, avec sa méthodologie proche de celle des sciences historiques : recherche, vérification et classification des sources, attention portée aux manuscrits, analyse du texte en fonction de critères extérieurs ${ }^{37}$. Or, Thomas White était considéré comme un éminent spécialiste de cette nouvelle approche. En mécaniste convaincu, il avait une approche très rationnelle des phénomènes surnaturels ou miraculeux. Il ne partageait pas du tout le point de vue de Bacon selon lequel leur étude n'entrait pas dans le cadre de la philosophie naturelle, et avait proposé pour le livre de la Genèse une approche fondée sur le calcul mathématique pour évaluer la possibilité d'un déluge ${ }^{38}$. Il est donc peu probable que ce partisan d'une exégèse ancrée dans la raison ait, sauf à renier ses 
convictions scientifiques, adhéré à l'ambiance de merveilleux de The Life of Gregorio López. Pourtant, l'adaptation anglaise garde, outre un parfum désuet et suranné de sainteté, les traces d'une tradition littéraire tout à fait antérieure à 1638 suivant laquelle la notion de vérité ne pouvait être que dogmatique. Il est fort possible que le théologien anglais ait considéré que l'inscription du texte dans cette longue et familière tradition hagiographique pouvait garantir l'intérêt des lecteurs catholiques anglophones restés attachés à ce genre littéraire.

\section{D'un désert à l'autre}

Une thématique retient particulièrement l'attention : celle du sauvage. Dans The Life of Gregorio López, l'Indien mexicain occupe le rôle de l'athée, envers lequel l'Espagnol ne faisait d'ailleurs preuve d'aucun prosélytisme, les autochtones ne constituant pas un objet d'attention pour le catholique, dont la spiritualité, toute de contemplation, n'incluait pas de dimension missionnaire. Ermite et sauvages cohabitaient pacifiquement ; ces derniers, est-il précisé, acceptèrent sans difficulté aucune le nouvel arrivé $^{39}$. Le chapitre IV offre un développement plus élaboré sur cette question. La grande tolérance des Indiens envers l'étranger y est mise en parallèle avec l'intolérance des clercs de l'Église catholique de Mexico qui prirent des mesures disciplinaires contre leur coreligionnaire, auquel ils reprochaient de ne guère fréquenter les lieux de culte ${ }^{40}$. Par contraste, les sauvages, en reconnaissant d'emblée la qualité de la spiritualité de l'Européen, manifestaient une connaissance naturelle de son vrai destin - connaissance dont les représentants de l'Église n'étaient pas détenteurs, puisqu'ils allèrent jusqu'à accuser l'Espagnol d'hérésie ${ }^{41}$. L'implicite du message se dévoile à travers l'opposition établie entre les deux groupes : par le truchement des Indiens incroyants, l'auteur peut dire la vérité que la rigidité théologique des clercs ne peut plus percevoir, l'appartenance à une structure ecclésiale signifiant l'aveuglement de l'entendement. La critique, de fait, s'adresse aux ordres réguliers, car ce sont bien les dominicains qui insistaient pour que Gregorio López accepte de vivre en communauté, proposition qu'il refusa. La hiérarchie des vocations se voit ici affirmée, la relation de l'ermite à Dieu étant indirectement posée comme supérieure à celle qui unit le clerc régulier à son Créateur. La relation privilégiée entre le mystique et Dieu, la relative sacralisation de l'homme pieux qui en découlait, constituaient des thèmes récurrents du courant mystique rhéno-flamand et une source de conflits avec le courant cartésien rationaliste français. La figure exotique du sauvage, qui n'existe pas en soi, mais uniquement comme fonction critique par rapport à l'institution catholique, permet de rouvrir, pour le lecteur européen, protestant en particulier, un débat connu sur les différentes voies vers la perfection. Non seulement les Églises issues de la Réforme ne reconnaissaient aucune prééminence de rang à la voie monastique, mais elles la récusaient comme telle. En clair, aborder narrativement cette question théologique essentielle à travers une réalité du Nouveau Monde transmettait au lecteur de l'Ancien Monde le sentiment d'une altérité très familière.

La figure de l'ermite en son désert offrait, elle aussi, un miroir dans lequel des protestants anglais, mais aussi des jansénistes français pouvaient se reconnaître. Alors que la traduction de Thomas White paraissait, en 1638, Jean Duvergier de Hauranne (1581-1643), abbé de Saint-Cyran et chef de file du mouvement des Solitaires de PortRoyal, était emprisonné. Cette même année mourait Jansénius. L'adaptation du 
théologien catholique anglais, ami de longue date de Port-Royal, par la caution qu'elle apportait à une conception de la vie religieuse à l'écart des structures ecclésiales et des ordres réguliers, constituait un acte militant de soutien à ces amis de France dont l'isolement politique, dès lors que le groupe fut dissous, en 1661, pouvait laisser craindre la disparition. Il n'est alors pas surprenant que, quelques lustres plus tard, en 1674, quand le jansénisme eut à nouveau le vent en poupe, La Vida de López ait été traduite par l'un des Solitaires les plus connus : Robert Arnauld d'Andilly (1589-1674) ${ }^{42}$. Il conviendrait, bien évidemment, de mener une analyse comparée de ces deux adaptations $^{43}$ qui participaient l'une comme l'autre d'une volonté marquée de perpétuer la promotion d'une tradition ascétique originale.

\section{Conclusion}

Le succès éditorial de The Life of Gregorio López ne tient pas à la qualité intrinsèque, littéraire ou théologique de l'œuvre espagnole, mais aux adaptations nuancées du traducteur, prenant en compte, dans tous leurs détails, les exigences religieuses et politiques des milieux de réception anglais et français. En d'autres termes, les lectorats, qu'ils aient été «blackloïstes", catholiques plus classiques, ou protestants, présupposaient des critères de réceptivité qu'il convenait de respecter afin de conférer la crédibilité requise à la figure de ce « saint » et au modèle de piété qu'il incarnait. Le saint n'existe pas en soi ; n'existe qu'une représentation de cette figure, elle-même remodelée par les milieux de réception qui lui donnent vie et crédibilité. Dans le cas de la traduction de Thomas White de 1638, cette remodélisation s'éloigne des canons de sainteté de l'orthodoxie romaine, tant par la mise en scène d'une spiritualité détachée de la figure du Christ et ancrée dans la mystique rhénane que par le choix de thématiques jansénistes. Toutefois, pour des lectorats peu au fait des controverses théologiques des années 1630, la présentation neutre de la piété et l'insistance sur des topoï médiévaux constituaient la garantie d'une position non partisane, puisque ancrée dans une longue tradition hagiographique. Cette garantie se voyait confortée par l'évocation d'un saint étranger, distant des polémiques du vieux continent, tant au sens littéral qu'au sens symbolique. Pourtant, au second niveau, étant donné l'engagement politico-religieux des traducteurs anglais et français férus de questions théologiques, l'adaptation relève d'un acte militant. L'image recomposée du saint cautionne alors les propres choix théologiques tant des jansénistes que des Blackloists. Il serait donc erroné d'interpréter ces succès d'édition comme des signes précurseurs d'un œcuménisme. Ceux qui s'étaient éloignés ou séparés de l'Église catholique, dont ils récusaient l'opposition dualiste entre orthodoxie et hérésie, affirmaient, par leur dynamisme éditorial, une volonté déterminée de redéfinir leur propre modèle de sainteté tout en réussissant à ne pas se couper d'une tradition hagiographique forcément catholique. 


\section{NOTES}

1. La Vida que hizo el siervo de Dios Gregorio Lòpez en algunos lugares de esta nueva Espana, biographie publiée à Madrid en 1613, fut rédigée par un moine, Francisco de Losa, compagnon de G. Lòpez. L'ouvrage parut la même année à Mexico; son original est conservé à la Biblioteca Historica de Madrid. Il connut cinq réimpressions : deux à Lisbonne, en 1615 et en 1625 ; une à Séville en 1618 ; deux à Madrid en 1618 et en 1624. Plusieurs variantes virent le jour : celle de Remon, dès 1613 ; celle de Munoz, à Madrid en 1630. Pour une liste complète des éditions en espagnol, en Nouvelle Espagne et dans la péninsule ibérique, voir: Antonio RUBIAL GARCIA, La Santidad Controvertida, Mexico : Universidad de Mexico, 1999, pp. 114-116. Je tiens à remercier Christopher Pedley, responsable de la bibliothèque de Heythrop College, University of London, pour avoir mis à ma disposition ces textes et m'avoir fait part de ses connaissances détaillées des archives catholiques anglaises du XVII ${ }^{\mathrm{e}}$ siècle.

La première traduction en français fut celle du jésuite Louis Conart : La Vie de Grégorio López dans la Nouvelle Espagne, Paris : chez Jean Henault, 1644. En 1674, le janséniste Robert Arnauld d'Andilly (1589-1674) proposa une deuxième traduction : La vie du bienheureux Grégoire López, Paris : Le Petit, 1674.

Pierre Poiret (1646-1719) édita la biographie de López en 1701 à Cologne, et lui donna pour titre Le Chrétien réel. Cette deuxième édition connut une vaste diffusion dans toute l'Europe. Poiret préfaça également une réédition en 1717.

La première édition anglaise, objet de la présente étude, parut en 1638 : Francisco de LOSA, The Life of Gregorio Lopez, translated by N.N.[Thomas White], Paris : Chez la veuve Blageard, 1638. Abraham Woodhead (1609-1678), un oratorien anglais, réalisa une deuxième traduction en anglais en 1675 (rééd. 1686), sur laquelle John Wesley (1703-1791), le fondateur du méthodisme, se basa pour sa propre adaptation. Voir Jean ORCIBAL, «Les spirituels français et espagnols chez John Wesley et ses contemporains ", Revue de l'Histoire des Religions, vol. 139, n 139-1, 1951, pp. 50-109.

2. Voir Jean-Michel SALLMAN, Naples et ses saints à l'âge baroque, Paris : PUF, 1994, pp. 64-68, 159-165.

3. L'ouvrage de référence sur les débats en Angleterre et en France sur ces questions demeure Georges TAVARD, La Tradition au XVII siècle en France et en Angleterre, Paris : Éditions du Cerf, 1969.

4. Jean CALvin, Traité des reliques, Genève : Pierre de La Rovière, 1599. Pour un commentaire du sermon de 1522 de Calvin, voir Jean DELUMEAU, « Les Réformateurs et la superstition », in Actes du colloque "l'Amiral de Coligny et son temps » (Paris, 24-28 octobre 1972), Paris : Société de l'histoire du protestantisme français, 1974, p. 455.

5. Pierre GIBERT, L'invention critique de la Bible, XV ${ }^{e}$-XVIII ${ }^{e}$ siècle, Paris : Gallimard, 2010.

6. LOSA, The Life of Gregorio López translated by N. N.[Thomas White].

7. Samuel halKeTt \& John LAING (eds.), A Dictionary of Anonymous and Pseudonymous Publications in the English Language, 1475-1640, London: Longman, 1980, $3^{\text {rd }}$ ed., 1980, p. 80. En réalité, la liste non exhaustive des pseudonymes de Thomas White incluait également: "Candido ", "Vitus", "Leblanc », « Bianchi »; ses surnoms n'étaient pas moins nombreux : «Backlot », " Baquelau », «Baguelar», «Blaqeau». Voir Ruth CLARK, Strangers and Sojourners at Port Royal, Cambridge: Cambridge University Press, 1932, pp. 10-11.

8. ${ }^{8}$ Bruno NEVEU, L'Erreur et son juge: Remarques sur les censures doctrinales à l'époque moderne, Napoli : Bibliopolis, 1993, pp. 310-322, 371-381 ; Beverley C. southGATE, Covetous of Truth: The Life and Work of Thomas White (1593-1626), Dordrecht: Kluwer, 1993 ; Jeffrey collins, 'Thomas White and the Backloist Conspiracy of 1649', The Historical Journal, vol. 45, n 2, 2000, pp. 305-331; Stefania 
TUTINo, Thomas White and the Blackloists: Between Politics and Theology during the English Civil War, Aldershot: Ashgate, 2008.

9. Thomas WHITE, Peripateticall Institutions, 1636 ; SOUTHGATE, op. cit., pp. 93-103.

10. TUTINO, op. cit, passim.

11. Adrien BAILlet, La Vie de Monsieur Descartes, 2 vols., Paris : Chez Daniel Horthemels, 1691, vol. 2 (liv. 7, chap. 3), p. 245 ; WHITE, Peripateticall Institutions ; SOUTHGATE, op. cit., pp. 93-103 ; tUTINO, op. cit, passim.

12. Thomas WHITE, The Grounds of Obedience and Government, 1655.

13. La liste des ouvrages de Thomas White censurés ou mis à l'index est donnée dans Pierre BAYLE, Dictionnaire historique et critique, 4 vols., Amsterdam : Chez P. Brunel, 1730, article « Anglus », vol. 1, pp. 238-239.

14. SOUTHGATE, op. cit., pp. 42-53.

15. Thomas WHITE, Sonus buccinae, sive Tres tractatus de virtutibus fidei et theologiae, de principiis earundem et de erroribus oppositis, authore Thoma Anglo ex Albiis East-Saxonum, 1654. Voir également, pour une analyse critique, BAYLE, op. cit. , pp. 238-239.

16. Sur cette extrême rareté des positions hérétiques selon Thomas White, voir NEVEU, op. cit., pp. 310-322, 371-381.

17. Pour une analyse détaillée des apports de Thomas White à la réflexion sur la Tradition, voir TAVARD, op. cit., pp. 396-438.

18. ANON. (ed.), Tracts Relating to Thomas White [1657], Douai, 1660.

19. Ibid.

20. NEVEU, op. cit. , pp. 310-322, 371-381.

21. Article « Thomas White », in Jean LESAULNIER \& Antony McKENNA (eds.), Dictionnaire de PortRoyal, Paris : Honoré Champion, 2004, pp.1017-1018.

22. Donald Goddard wiNG (ed.), Short-Title Catalogue of Books Printed in England, Scotland, Ireland, Wales and British America and of English Books Printed in Other Countries, 1641-1700, 3 vols., $2^{\text {nd }}$ ed., New York: Modern language Association of America, 1988, vol. 3, pp. 693-694. Voir également HALKETT \& LAING, op. cit., C 39, D 139.

23. Voir à ce sujet Thérèse-Marie JALLAIS, "L'étrange cathéchisme méthodiste ", Revue d'histoire et de philosophie religieuse, vol. 90, $\mathrm{n}^{\circ}$ 3, juillet-septembre 2010, pp. 345-351.

24. LOSA, The Life of Gregorio López translated by N. N.[Thomas White], p. 4.

25. Il n'était pas rare, en pleine Contre-Réforme, que les mystiques soient suspectés d'avoir un passé religieux, personnel ou familial peu orthodoxe. Voir Michel de CERTEAU, La Fable mystique: XVI ${ }^{e}$-XVII ${ }^{e}$ siècle, Paris : Gallimard, 1982, p. 38. Pour ce qui est de Thérèse d'Avila, voir Julia KRISTEVA, Thérèse mon amour, Paris : Fayard, 2008, passim.

26. LOSA, The Life of Gregorio López translated by N. N.[Thomas White], pp. 20-27, 40-44.

27. Ibid. et pp. 15-19.

28. ${ }^{28}$ Benoît de CANFELD, Reigle de perfection, Paris : Veuve Charles Chastellain, 1621-1622.

29. William Reginald WARD, Early Evangelicalism, Cambridge: Cambridge University Press, 2006, pp. 1-5, 40-69.

30. LOSA, The Life of Gregorio López translated by N. N [Thomas White], pp. 4-13.

31. Ibid.

32. ATHANASE D'ALEXANDRIE, Vie d'Antoine, Paris : Éditions du Cerf, 1994.

33. On retrouve ces mêmes éléments dans Alonso RAmos, op. cit., lib. 1, cap. 1, vol. 1, fol. 3.

34. LOSA, The Life of Gregorio López translated by N. N.[Thomas White], pp. 17-27.

35. Ibid. et LOSA, La Vida que hizo el siervo de Dios Gregorio López en algunos lugares de esta nueva España, pp. 343-345, 367, passim.

36. Ibid., cap. 8. 
37. Daniel Haperbrach et d'autres Bollandistes éditèrent, à partir des années 1643, des travaux érudits. Voir GIBERT, op. cit., passim.

38. SOUTHGATE, op.cit., p. 133.

39. LOSA, The Life of Gregorio López translated by N. N.[Thomas White], chap. IV.

40. Ibid., p. 92.

41. Ibid., p. 44.

42. Robert Arnaud d'ANDILly, La vie du bienheureux Grégoire López, Paris : Le Petit, 1674.

D’Andilly avait déjà traduit des ouvrages de spiritualité érémitique, dont Les Vies des Saints Pères des déserts (Paris : Le Petit, 1647) et une œuvre de Saint Jean Climaque, L'Échelle sainte ou les degrés pour monter au ciel (Paris : Le Petit, 1658).

43. Dans l'adaptation de Robert Arnaud d'Andilly, les différentes étapes de la vie de López suivent les temps de l'échelle spirituelle du paradis de Climaque : rupture avec le monde, recherche des vertus fondamentales, lutte contre les passions, insistance sur le quotidien dans sa dimension pratique.

\section{RÉSUMÉS}

La Vida de Gregorio López (1613) connut de nombreuses réécritures et rééditions dans les milieux de la Réforme en Europe. L'étude de l'adaptation anglaise du catholique Thomas White (1592-1676) permet d'expliquer ce surprenant succès. Ce texte de 1638 reste ancré dans la tradition hagiographique médiévale, mais dépeint une spiritualité de type rhéno-flamand, et insiste sur la thématique du désert. Est ainsi remodélisée une figure de sainteté peu orthodoxe, mais acceptable dans les milieux protestants et catholiques « blackloïstes » en Angleterre comme dans les cercles jansénistes en France.

La Vida de Gregorio Lòpez (1613) went through many different editions in Protestant countries in Europe. The study of its 1638 English adaptation by the Catholic Thomas White (1592-1676) may well explain this surprising success. The text is anchored in the hagiographic medieval tradition, but it depicts a Rhino-Flemish type of spirituality and puts a strong emphasis on the theme of the desert. The new unorthodox model of sainthood was made acceptable to Protestants and Blackloists in England as well as to Jansenists in France.

\section{AUTEUR}

\section{THÉRÈSE-MARIE JALLAIS}

Université François Rabelais (Tours) 